\title{
Management ATG (SIRS)
}

\author{
Francesca Bonifazi
}

\subsection{Introduction}

Currently horse and rabbit anti-lymphoglobulins (ATLG) or antithymocyte globulin (ATG) is available; the main, although not exclusive, use is for the treatment of aplastic anemia (horse) and for GVHD prophylaxis (rabbit). They differ in the manufacturing process (i.e., used animal, pulsed antigens, antibody specificities, and cellular targets): for this reason, dose, timing, and setting cannot be interchangeable, and also clinical results are different. As they are polyclonal serum-derived products from nonhuman organisms, they can cause serum sickness and infusion reactions.

\subsection{ATLG/ATG Infusion Protocol (See Table 26.1)}

ATLG/ATG infusion should be performed in trained centers. Standard hygienic handling of the injection site, careful evaluation of the infusion speed, and appropriate choice of the venous access are crucial. Medical personnel should carefully watch over patients for adverse events not only during but also after infusion.

\section{F. Bonifazi $(\bowtie)$}

Institute of Hematology "Seràgnoli”, University

Hospital S. Orsola-Malpighi, Bologna University,

Bologna, Italy

e-mail: francesca.bonifazi@unibo.it
During administration, the patient needs to be monitored for symptoms related to infusion reactions or anaphylaxis. The first dose should be administered at a reduced speed for the first $30 \mathrm{~min}$. If no symptoms of intolerance occur, infusion rate may be increased. In case of anaphylactic or anaphylactoid reactions, physicians must be prepared to promptly manage this event, and appropriate medical treatment has to be implemented.

A central venous catheter is preferred, although a peripheral large high-flow access may be acceptable, if a central line is not available. Thrombophlebitis is the major risk when a peripheral vein is used. The availability of a highflow access is important in case of treatment of infusion reactions.

Premedication is mandatory in order to improve systemic and local tolerance (see later). Stability, compatibility, and dilution are different for each product, and specific manufacturer recommendations should be followed carefully.

Preinfusion intraepidermal tests are not yet validated for rabbit ATG but, according to manufacturer indications, are recommended for horse ATLG.

- Although standard infusion time is between 4 and $12 \mathrm{~h}$, a longer administration time correlates with milder side effects, thus making infusions of $12 \mathrm{~h}$ highly advised. 
Table 26.1 Infusion of ATLG/ATG

\begin{tabular}{|l|l|}
\hline Factors & Comments \\
\hline Infusion site & - Central line is highly preferred \\
& - Risk of thrombophlebitis and drug precipitation are higher in peripheral \\
& veins
\end{tabular}

\subsection{ATLG/ATG Dose}

Dose and timing of ATLG/ATG administration vary substantially among transplant centers (Bacigalupo et al. 2001; Finke et al. 2009; Walker et al. 2016; Kröger et al. 2016; Soiffer et al. 2017).

The currently used doses of ATLG/ATG are calculated and validated in clinical trials, according to body weight. A strong rationale and some preliminary data (Admiraal et al. 2017) suggest that calculating the ATG/ATLG dose according to the cellular target, i.e., the number of total lymphocyte before infusion of the first dose, can provide the optimal drug exposure and therefore maximize the benefit (GVHD decrease) over the potential risks (increase of relapses and infections). Since ATLG and ATG are different preparations arising from different manufacturing processes and different pulsed antigens, no dose equivalence can be established.

\subsection{Infusion Reactions}

ATLG/ATG administration can be complicated by several infusion reactions including fever, chills, erythema, dyspnea, oxygen desaturation, nausea/vomiting, diarrhea, abdominal pain, hyperkalemia, tachycardia, hypo- or hypertension, malaise, rash, urticaria, headache, arthralgia, myalgia (serum sickness, after 5-15 days from infusion), hepatic cytolysis, and even systemic anaphylaxis.

Even if the NCI Terminology Criteria for Adverse Events (CTCAE) use different scales for grading reactions to infusion of chemotherapy and allergic/anaphylaxis reactions, there are no specific symptoms enabling to distinguish "standard" infusion reaction from an allergic one that can evolve to anaphylaxis.

Anaphylaxis and acute allergic reactions are based on IgE effect and histamine release by mastocytes, but the vast majority of symptoms can be 
attributed to the cytokine release syndrome (CRS) and are generally reversible. CRS is a form of systemic inflammatory response syndrome (SIRS) (Matsuda and Hattori 2006; Balk 2014). CRS can follow not only ATG/ATLG infusion but also chemotherapy, MoAb (Remberger et al. 1999; Feng et al. 2014), bispecific antibodies, or CAR-T cell therapies (Lee et al. 2014). All these (both allergic and nonallergic, such as CRS) are infusion reactions. Serum sickness is a hypersensitivity phenomenon that can develop after 5-15 days after the infusion, and it is well responsive to steroid treatment.

\subsection{SIRS}

SIRS is a clinical syndrome due to dysregulated inflammation. SIRS may occur in several conditions, such as infection, autoimmune disorders, vasculitis, thromboembolism, chemotherapy infusion, surgery, and burns. The denomination originates from changes of some parameters (temperature, heart and respiratory rates, and white blood cell count) occurring after infection/ sepsis according to Bone (Bone et al. 1992). A pediatric version tailored on patient age is also available (Goldstein 2005). More recently, some authors (Lee et al. 2014) revised the classification of the cytokine release syndrome according to the treatment required (oxygen, vasopressors, organ toxicity).

\subsubsection{Risk Factors for SIRS}

SIRS after ATLG/ATG infusion cannot be predicted and the risk factors are not well known.

The binding of ATLG/ATG to the surface of target cells (lymphocytes, monocytes, dendritic cells) elicits cytokine production and systemic inflammation (Bone et al. 1992).

Thus, that the higher the number of lymphocytes at the moment of the (first) infusion, the more likely is the risk of systemic activation of inflammation and then SIRS.
Accordingly, RIC regimens are reported to be associated with greater cytokine release syndrome (Remberger and Sundberg 2004) because of the likely higher number of residual lymphocytes in RIC in comparison with MAC regimens.

\subsubsection{Management of SIRS}

\subsubsection{Prophylaxis}

ATLG/ATG infusion reactions can be reduced in frequency and severity by two factors: premedication and speed of infusion. Premedication is performed with steroids, antihistamine, and acetaminophen. The optimal schedule of premedication is not yet well established. Doses of prednisolone of $250 \mathrm{mg}$ (higher than $1 \mathrm{mg} / \mathrm{kg}$ ), given before the first infusion and followed by an additional dose in the same day, reduce the incidence of infusion reactions and cytokine release as reported (Pihusch et al. 2002).

The rate of infusion is one of the most important factors to reduce the incidence and severity of infusion reactions since lower infusion rates are associated with a lower incidence and the severity of reactions. Administration time $\geq 12 \mathrm{~h}$ is the preferred schedule to yield high compliance to ATLG/ATG infusion.

\subsubsection{Treatment}

If symptoms of SIRS appear, the drug should be discontinued, at least temporarily.

Treatment is symptomatic and depends upon the clinical manifestations. Intensive care for respiratory and hemodynamic support should be given according to international guidelines for critical patients, and the intervention of an intensive specialist may be requested. SIRS after ATLG/ATG is different from sepsis-induced SIRS where steroids failed to achieve a significant benefit (Cronin et al. 1995). Symptoms due to ATLG/ATG-related SIRS are more pronounced on day +1 and then tend to decrease. Steroids, widely used preemptively, provide high response rates also as a treatment measure. 


\section{Permanent Discontinuation/Rechallenge}

Rechallenge after anaphylaxis and after standard infusion reactions $>3$ is strongly discouraged.

Non-controlled life-threatening infections are contraindications to transplant and should not modify ATLG/ATG administration per se.

Desensitization protocols are not yet clearly validated.

\section{Key Points}

- SIRS is a systemic reaction related to cytokine release after ATLG/ATG infusion.

- The infusion reactions can be reduced by premedication (steroids, antihistamines, and acetaminophen) and by a low infusion rate ( $12 \mathrm{~h}$ or longer).

\section{References}

Admiraal R, Nierkens S, de Witte MA, et al. Association between anti-thymocyte globulin exposure and survival outcomes in adult unrelated haemopoietic cell transplantation: a multicentre, retrospective, pharmacodynamic cohort analysis. Lancet Haematol. 2017;4:e183-91.

Bacigalupo A, Lamparelli T, Bruzzi P, et al. Antithymocyte globulin for graft-versus-host disease prophylaxis in transplants from unrelated donors: 2 randomized studies from Gruppo Italiano Trapianti Midollo Osseo (GITMO). Blood. 2001;98:2942-7.

Balk RA. Systemic inflammatory response syndrome (SIRS): where did it come from and is it still relevant today? Virulence. 2014;5:20-6.

Bone RC, Balk RA, Cerra FB, et al. American College of Chest Physicians/Society of Critical Care Medicine Consensus Conference: definitions for sepsis and organ failure and guidelines for the use of innovative therapies in sepsis. Crit Care Med. 1992;20:864-74.

Cronin L, Cook DJ, Carlet J, et al. Corticosteroid treatment for sepsis: a critical appraisal and meta-analysis of the literature. Crit Care Med. 1995;23:1430-9.
Feng X, Scheinberg $\mathrm{P}$, Biancotto $\mathrm{A}$, et al. In vivo effects of horse and rabbit antithymocyte globulin in patients with severe aplastic anemia. Haematologica. 2014;99:1433-40.

Finke J, Bethge WA, Schmoor C, et al. Standard graftversus-host disease prophylaxis with or without anti-T-cell globulin in haematopoietic cell transplantation from matched unrelated donors: a randomised, open-label, multicentre phase 3 trial. Lancet Oncol. 2009;10:855-64.

Goldstein B, Giroir B, Randolph A, International Consensus Conference on Pediatric Sepsis. International pediatric sepsis consensus conference: definitions for sepsis and organ dysfunction in pediatrics. Pediatr Crit Care Med. 2005;6:2-8.

Kröger N, Solano C, Wolschke C, et al. Antilymphocyte globulin for prevention of chronic graft-versus-host disease. N Engl J Med. 2016;374:43-53.

Lee DW, Gardner R, Porter DL, et al. Current concepts in the diagnosis and management of cytokine release syndrome. Blood. 2014;124:188-95.

Matsuda N, Hattori Y. Systemic inflammatory response syndrome (SIRS): molecular pathophysiology and gene therapy. J Pharmacol Sci. 2006;101:189-98.

Pihusch R, Holler E, Mühlbayer D, et al. The impact of antithymocyte globulin on short-term toxicity after allogeneic stem cell transplantation. Bone Marrow Transplant. 2002;30:347-54.

Remberger M, Sundberg B. Cytokine production during myeloablative and reduced intensity therapy before allogeneic stem cell transplantation. Haematologica. 2004;89:710-6.

Remberger M, Svahn BM, Hentschke P, et al. Effect on cytokine release and graft-versus-host disease of different anti-T cell antibodies during conditioning for unrelated haematopoietic stem cell transplantation. Bone Marrow Transplant. 1999;24:823-30.

Soiffer RJ, Kim HT, McGuirk J, et al. Prospective, randomized, double-blind, phase III clinical trial of antit-lymphocyte globulin to assess impact on chronic graft-versus-host disease-free survival in patients undergoing HLA-matched unrelated myeloablative hematopoietic cell transplantation. J Clin Oncol. 2017;35:4003-11.

Walker I, Panzarella T, Couban S, et al. Pretreatment with anti-thymocyte globulin versus no anti-thymocyte globulin in patients with haematological malignancies undergoing haemopoietic cell transplantation from unrelated donors: a randomised, controlled, open-label, phase 3, multicentre trial. Lancet Oncol. 2016;17:164-73. 
Open Access This chapter is licensed under the terms of the Creative Commons Attribution 4.0 International License (http://creativecommons.org/licenses/by/4.0/), which permits use, sharing, adaptation, distribution and reproduction in any medium or format, as long as you give appropriate credit to the original author(s) and the source, provide a link to the Creative Commons license and indicate if changes were made.

The images or other third party material in this chapter are included in the chapter's Creative Commons license, unless indicated otherwise in a credit line to the material. If material is not included in the chapter's Creative Commons license and your intended use is not permitted by statutory regulation or exceeds the permitted use, you will need to obtain permission directly from the copyright holder.

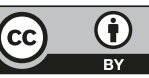

